\title{
Linx
}

Revue des linguistes de l'université Paris X Nanterre

$53 \mid 2005$

Le semi-figement

\section{Expression compositionnelle ou locution : ça craint vs ça barde?}

Marie-Pierre Sales

\section{CpenEdition}

Journals

Édition électronique

URL : http://journals.openedition.org/linx/304

DOI : $10.4000 / \operatorname{linx} .304$

ISSN : 2118-9692

Éditeur

Presses universitaires de Paris Nanterre

Édition imprimée

Date de publication : 1 décembre 2005

ISSN : 0246-8743

Référence électronique

Marie-Pierre Sales, «Expression compositionnelle ou locution : ça craint vs ça barde? », Linx [En ligne], 53 | 2005, mis en ligne le 15 février 2011, consulté le 03 mai 2019. URL : http://

journals.openedition.org/linx/304; DOI : 10.4000/linx.304 


\title{
Expression compositionnelle ou locution : ça craint vs ça barde?
}

\author{
Marie-Pierre Sales \\ Université de Paris $X$ et Laboratoire MoDyCo (CNRS UMR \\ 7114)
}

\section{Introduction}

Si l'on relève des énoncés dans lesquels le pronom ça apparaît en position de sujet, on s'aperçoit que beaucoup d'expressions comme ça y est, ça craint, ça va, ça barde, sont qualifiées, à tort ou à raison (c'est ce que nous tenterons de déterminer), de figées ou d'idiomatiques. Pourquoi de telles expressions peuvent-elles être assimilées à des locutions ? Que recouvre l'idée de figement quand on prend en compte ces énoncés?

Lorsque l'on parle d'expressions verbales figées, ou de locutions verbales, on fait généralement référence à un verbe accompagné d'un syntagme nominal. Le verbe et le syntagme nominal forment une locution dont le sens n'est pas la somme de ses parties. Ou, pour reprendre la définition de Gaston Gross (1996:14) : « on pourrait appeler locution tout groupe dont les éléments ne sont pas actualisés individuellement ». Selon cette définition, une locution a un sens qui n'est pas transparent comme c'est le cas pour une expression dite compositionnelle. Toute phrase compositionnelle est le produit des éléments qui la constituent; son sens dépend d'un prédicat et des arguments qui lui sont associés. 
Contrairement aux phrases compositionnelles, qui forment la grande majorité de la parole, on peut citer quelques exemples de locutions verbales : casser sa pipe, prendre le taureau par les cornes, ou de phrases figées: les carottes sont cuites, la moutarde me monte au nez... Or, s'il y a une opacité sémantique (pour reprendre le terme de G. Gross), qui justifie l'appellation de locution, les conséquences syntaxiques sont multiples. Les locutions n'acceptent pas en effet les transformations syntaxiques qui s'appliquent d'ordinaire à une phrase. En effet, aucune des expressions citées ci-dessus ne peut autoriser l'insertion d'un modifieur (comme un adjectif ou un adverbe) devant le nom. De plus, le déterminant, le verbe ou le nom ne peuvent commuter avec aucun élément de la même classe sur l'axe paradigmatique. De telles expressions sont à interpréter comme une seule unité lexicale. Leur analyse relève à la fois de la sémantique (puisque le sens de chaque terme se perd dans la signification d'ensemble de l'expression) et de la syntaxe (puisqu'elles ne peuvent pas admettre de transformations syntaxiques comme la pronominalisation, la passivation...). Ce qui nous intéresse plus particulièrement dans cet article, ce sont les locutions dont c'est le syntagme nominal sujet qui est figé. Or, il apparait que la grammaire générative a souvent peu fait état du figement d'une séquence sujet-verbe $(\mathrm{GN} \mathrm{GV})$ au profit des séquences verbe-objet $(\mathrm{V}$ GV). Pourtant, nous dirons qu'un sujet, comme un objet, peut être figé quand le syntagme nominal ne peut subir aucune modification syntaxique.

Mais comment rapprocher une séquence comme les carottes sont cuites d'expressions qui utilisent ça comme sujet et que l'on qualifie de figées ? La comparaison soulève un problème de taille puisque ça est un pronom. Or, comment postuler qu'un pronom (comme ca) appartienne à une locution au même titre qu'un GN ? Par quels tests le démontrer ? Y a-t-il figement ou semi-figement de l'expression? Nous essaierons de répondre à ces questions en établissant des distinctions entre certaines expressions qui utilisent $c ̧ a$ comme sujet. Puis nous verrons que l'interprétation différente que l'on peut faire de ça comme sujet (anaphorique, quasi impersonnel ou figé) a des conséquences à la fois syntaxiques et lexicologiques.

\section{1. Ça comme sujet d'expressions : quatre cas à distinguer}

Au sein des expressions qui utilisent ça comme sujet et que l'on qualifie de figées, des différences sont à effectuer. En effet, si l'on utilise les tests de la commutation et de la dislocation, on peut établir un classement des expressions, d'après le rôle que joue ça.

\subsection{Une commutation parfaite (ça (me) fait froid dans le dos vs ça barde)}

Si l'on compare des expressions comme ça fait froid dans le dos et ça barde, on perçoit immédiatement une différence: dans la première, ça peut commuter avec n'importe quel autre sujet sans que le sens de la locution verbale ne change; dans le second, il ne peut être remplacé par aucun syntagme, sous peine d'agrammaticalité :
a. ça fait froid dans le dos
b. cette histoire fait froid dans le dos
c. ça barde à côté
d. * la pièce à côté barde 
e. ça rime à rien

f. ton inquiétude rime à rien

On peut donc dire que ce n'est pas tant ça fait froid dans le dos qui est une locution verbale que faire froid dans le dos. La conséquence est que, dans cette locution, ça n'est qu'un sujet possible, parmi beaucoup d'autres, et rien ne permet d'en faire un sujet privilégié, si ce n'est, peut-être, une fréquence d'utilisation plus élevée, constatée dans les corpus. La locution, formée de constituants qui n'ont plus de sens individuellement, est alors synonyme d'une unité lexicale simple ; dans ce cas, la locution faire froid dans le dos est synonyme de effrayer.

On peut faire la même analyse pour ça rime à rien puisque, dans cette locution, un autre sujet que ça est possible, comme le montre l'exemple (1f).

La structure d'une locution en plusieurs constituants pourrait poser le problème du classement des locutions dans un dictionnaire. A quelle entrée vont-elles figurer? La question est résolue depuis longtemps par des dictionnaires spécialisés qui classent les locutions à une entrée, en faisant des renvois aux entrées de toutes les composantes pleines de la locution. De ce point de vue, Le dictionnaire d'expressions et locutions de Rey et Chantreau (1997) confirme notre idée que ça n'est qu'un sujet possible de faire froid dans le dos puisque la locution n'a d'entrée pour aucun sujet spécifique.

\subsection{Une commutation possible mais forcée (ça me fait une belle jambe)}

Le deuxième cas nous semble légèrement différent puisque, à travers l'exemple de ça me fait une belle jambe, on s'aperçoit que la commutation de ça avec un autre syntagme reste possible mais qu'elle semble beaucoup moins naturelle que dans le premier cas et paraît même un peu forcée :

(2) a. ça me fait une belle jambe

b. ton histoire me fait une belle jambe

c. ton histoire, ça me fait une belle jambe

d. tes soucis me font une belle jambe

e. tes soucis, ça me fait une belle jambe

f. tes soucis, ils me font une belle jambe

Les exemples (2) montrent que si l'acceptabilité des expressions ne fait aucun doute, la reprise du syntagme nominal par ça, dans le cadre d'une dislocation, est préférable à la seule commutation. La commutation est possible mais la dislocation, permettant un système de reprise (par ça ou un pronom personnel), est meilleure.

\subsection{Une expression semi-figée ? (ça craint, ça fait rien)}

Le troisième cas, quant à lui, montre un premier degré de figement de l'expression. C'est G. Gross (1996 : 16) qui a explicité la notion de degré de figement que nous reprenons :

dans une chaîne donnée, une partie seulement de l'ensemble peut faire l'objet d'un figement, tandis que le reste relève d'une combinatoire libre. (...) Pour exprimer la 
Marie-Pierre Sales

notion de 'bonne cuisinière', on ne peut dans cordon-bleu substituer à cordon aucun autre substantif ni aucun adjectif à bleu. On peut en dire autant de fait divers. (...) Nous avons affaire ici à un figement qu'on pourrait appeler total. [...] Mais cette situation n'est pas la plus fréquente. On trouve souvent, dans une position donnée, une possibilité de paradigme. Ainsi, dans la suite rater le coche, on peut remplacer le verbe rater par louper ou manquer. Il y a là une liberté lexicale, même si le sens reste opaque dans les trois cas. [...] les possibilités de commutation sont plus ou moins importantes ; l'absence de paradigme n'est qu'un cas limite.

Si l'on applique cette idée de degré aux expressions qui utilisent ça comme sujet, on peut percevoir un premier degré de figement lorsque la commutation avec un autre sujet n'est quasiment plus possible ou apparaît très maladroite. C'est ce que font apparaitre les exemples suivants :

(3) a. ?? ton travail craint

b. ton travail, ça craint / ça craint, ton travail

c. ?? tes théories craignent

d. tes théories, ça craint

e. tes théories, elles craignent

Ainsi l'exemple (3a) est à la limite de l'acceptabilité, pour certains locuteurs, tandis que l'exemple (3b), mettant en place une dislocation droite ou gauche, pallie le problème de l'acceptabilité. Il existe néanmoins toujours un co-référent à ça, visible grâce à la dislocation, ce qui ne sera plus le cas pour des expressions comme ça barde. En fait, il est important de préciser qu'il n'est pas tout à fait juste de parler de coréférence avec ça.

Le pronom démonstratif, au contraire d'un pronom personnel ( $3 \mathrm{~d}$ vs $3 \mathrm{e}$ ), ne reprend pas seulement le contenu référentiel d'un syntagme nominal mais tout un implicite qui lui est attaché. Corblin (1995 : 90 et sq.) parle à propos de cela d'un pronom à référent indistinct non délimité. Cette propriété s'applique également à ça et justifie la réserve que nous faisons au fait de parler de co-référence :

les formes neutres ce, ceci, cela, s'emploient quand on n'a aucun nom dans l'esprit $[\ldots]$ il y a « une sorte d'expansion » métonymique du référent initial, et cela s'interprète comme mention d'un référent moins strictement délimité : de l'objet initial, on peut passer à la classe dont l'objet initial est le représentant [...] l'emploi de cela implique un référent non délimité, à contours flous...

On constate néanmoins que ça peut renvoyer à un nom identifiable dans le cotexte. C'est pourquoi on peut, pour ce troisième cas, parler de semi-figement; ce phénomène est illustré par des expressions comme ça craint, ça fait rien, ça le fait, ça me/te va, ça me/te dit. Encore faudrait-il préciser qu'une différence est à faire entre ça craint qui associe une seule lexie (craindre) au pronom et d'autres expressions (comme ça fait rien, ça le fait, ça me dit...) qui proposent des locutions verbales (constituées de plusieurs lexies) associées à ça. 


\subsection{Des locutions : ça barde, ça baigne, ça y est}

Pour ce quatrième cas, il semble difficile de faire subir des transformations syntaxiques à des expressions comme ça barde, ça baigne ou ça y est :

(4) a. ?? ton travail, ça baigne

b. * ton travail baigne

c. ça barde à côté

d. * la pièce à côté, ça barde

e. ton dossier, ça y est

f. * ton dossier y est

A travers ces exemples, on perçoit que la commutation de ça avec un autre syntagme n'est plus possible et que le détachement d'un syntagme et sa reprise par le démonstratif, dans le cadre d'une dislocation, peut être maladroit. L'exemple caractéristique est, de ce point de vue, ça barde, qui ne supporte ni la commutation, ni la dislocation. Les exemples (4a) et (4) montrent qu'une dislocation parait encore possible mais que la commutation, à la différence du cas (3) (ça craint) est impossible. Il n'est pas sûr, d'ailleurs, que (4a) et (4e) puissent s'analyser en terme de dislocation avec reprise pronominale (et donc dans une perspective co-référentielle). Ils s'interprètent plutôt comme un détachement du thème, sans que le pronom renvoie directement au syntagme.

La commutation et la dislocation permettent de faire apparaître des différences entre les expressions qui utilisent ça comme sujet, dont voici un tableau récapitulatif:

\begin{tabular}{|c|c|c|c|}
\hline $\begin{array}{c}1^{\mathrm{er}} \text { cas }: c ̧ a \\
\text { n'est qu'un } \\
\text { sujet possible }\end{array}$ & $\begin{array}{c}2^{\mathrm{e}} \text { cas }: \text { ça est un } \\
\text { sujet préféré à } \\
\text { d'autres }\end{array}$ & $\begin{array}{c}3^{\mathrm{e}} \text { cas }: \text { ça est un sujet } \\
\text { privilégié (semi- } \\
\text { figement) }\end{array}$ & $\begin{array}{c}4^{\mathrm{e}} \text { cas }: \text { ça est le seul } \\
\text { sujet possible } \\
\text { (locutions) }\end{array}$ \\
\hline $\begin{array}{c}\text { Commutation } \\
\text { parfaite de ça } \\
\text { avec un autre } \\
\text { sujet }\end{array}$ & $\begin{array}{c}\text { Commutation } \\
\text { possible mais } \\
\text { forcée } ; \\
\text { Dislocation } \\
\text { préférable }\end{array}$ & $\begin{array}{c}\text { Commutation très } \\
\text { maladroite } \\
\text { Dislocation possible }\end{array}$ & $\begin{array}{c}\text { Commutation } \\
\text { impossible } \\
\text { Dislocation } \\
\text { maladroite } \\
\text { dans certains } \\
\text { cas }\end{array}$ \\
\hline $\begin{array}{c}\text { ça fait froid } \\
\text { dans le dos } \\
\text { ça rime à rien }\end{array}$ & $\begin{array}{c}\text { ça fait une belle } \\
\text { jambe }\end{array}$ & $\begin{array}{c}\text { ça craint ; ça me dit } \\
\text { ça fait rien } \\
\text { ça le fait ; ça me va }\end{array}$ & $\begin{array}{c}\text { ça barde } \\
\text { ça baigne } \\
\text { ça y est }\end{array}$ \\
\hline
\end{tabular}

A la lecture de ce tableau, si l'on parle de semi-figement pour ça craint et de figement pour ça barde, comment est-il possible d'affiner la description de telles expressions? Quelles sont les propriétés spécifiques à rattacher à chacune d'elles ? 


\section{Description lexicographique de deux constructions : ça barde vs ça craint}

On ne peut vraiment parler de locution que dans le quatrième cas, pour des expressions comme ça y est, ça barde, ça baigne. Mais est-il possible d'aller plus loin dans la description et de trouver des propriétés communes à ces expressions, sur le plan sémantique comme sur le plan syntaxique?

\section{1. Ça barde, ça baigne... : locution et classe de verbe}

Le point commun qu'entretiennent ces expressions est celui de renvoyer, de façon générale, à la situation d'énonciation du locuteur. A travers de telles expressions, celui-ci qualifie son environnement. Les exemples qui suivent permettent d'en établir une liste succincte :

(5) a. ça barde
b. ça baigne
c. ça boume
d. ça gåe
e. ça repart
f. ça décolle

Nous proposons de réunir ces verbes sous l'appellation de verbes d'ambiance à partir d'un trait sémantique qu'ils partagent. Une distinction est cependant à faire entre deux types de verbes : ceux qui n'admettent que ça comme sujet (barder, gaze, boumer) et ceux qui autorisent d'autres sujets (baigner, repartir, décoller) mais qui, lorsqu'ils se combinent avec le démonstratif, ont le même objectif que les premiers : parler de l'environnement du locuteur. Le premier groupe de verbes, qui peut avoir pour origine une onomatopée (boumer) constitue une liste ouverte qui tend à s'élargir par la création de néologismes véhiculés par certains secteurs d'activités (comme, par exemple, la publicité).

Pour en revenir à la qualification, par le locuteur, de son environnement, on peut citer Corblin (1995 : 97) qui a insisté sur le lien existant entre le démonstratif et la situation d'énonciation, lien qui justifie l'utilisation fréquente d'indications spatiotemporelles en combinaison avec le démonstratif:

l'emploi de cela implique un référent non délimité, à contours flous, qui se confond avec la situation d'énonciation. Il faudrait reprendre en détail toutes les contraintes qui déterminent de tels énoncés, leur rapport privilégié à la sphère de l'énonciation, à l'exclamation notamment. Il semble cependant assez clair que ce tour réalise une sorte de dissolution du personnel dans la situation, d'où l'association privilégiée à des localisations spatiales ou temporelles [...] On comprend assez bien que ces localisations deviennent nécessaires si la forme mentionne un référent sans limites précisément assignables. 
Si le démonstratif renvoie à la situation d'énonciation, on comprend mieux alors pourquoi une classe de verbes (les verbes d'ambiance) qui utilisent uniquement ça comme sujet, se combinent bien souvent avec des indications spatio-temporelles. En effet, des exemples relevés sur Internet montrent que ces verbes s'accompagnent la plupart du temps d'une référence à un lieu ou à un destinataire :

(6) a. ça barde à Canal + / en Bretagne / dans la Sarthe / à Paris

b. ça baigne (pour le cinéma d'animation + pour eux)

c. ça boume pour iPod

Mais, au delà de cette homogénéité sémantique, ce type de construction en $c a$ $V$, que l'on peut qualifier de figée, repose sur un pronom démonstratif qui, au même titre que d'autres séquences figées ayant un GN pour sujet, ne peut pas subir de modifications syntaxiques. Nous allons à présent développer ce point.

\subsection{Un syntagme non modifiable au niveau syntaxique}

Si les locutions comme ça barde ou ça baigne ont des traits sémantiques communs, elles ont aussi un comportement syntaxique identique. En effet, ces expressions ne supportent l'insertion d'aucun modifieur comme aussi ou tout à rattacher à ça :

(7) a. * ça aussi barde

b. ?? ça aussi, ça barde

c. là aussi, ça barde

d. * tout ça barde

e. ? tout ça, ça barde

f. ça aussi, ça craint

g. tout ça, ça craint

D'après ce critère, le pronom sujet dans ça barde est non modifiable (contrairement à ça craint), tout comme l'est le sujet de séquences figées qui ont un sujet nominal. Rappelons qu'une séquence figée ne peut accepter la modification de son GN sujet comme le montrent les exemples (8):
a. les carottes sont cuites
b. ${ }^{*}$ tes carottes sont cuites
c. * les bonnes carottes sont cuites
d. * les carottes délicieuses sont cuites

Ces exemples prouvent que le syntagme nominal sujet d'une locution est figé, si le déterminant ne peut commuter avec aucun autre (8b), si l'insertion d'un modifieur comme un adjectif, avant (8c) ou après (8d) le nom est impossible ${ }^{1}$. L'insertion d'un modifieur rend inacceptable une séquence figée (cf. les exemples 8); comme c'est précisément le cas des exemples (7), nous pouvons alors percevoir le figement d'une

\footnotetext{
1 Pour un développement plus complet de la question, nous renvoyons à M. Gross (1982).
} 
structure ça $V$ sur le plan syntaxique (ce qui justifie notre rapprochement entre les exemples (7) et (8), qui pourrait paraittre inattendu).

Un second critère syntaxique permettant de voir que les locutions comme ça barde n'acceptent pas de transformations est le test de la négation. Là encore, une distinction est à faire entre ça barde et ça craint. Les verbes dits d'ambiance n'acceptent pas la négation qui est, en revanche, possible pour des expressions semi figées :

(9) a. * ça barde (pas + plus)

b. ça boume? non. * ça boume pas

c. * ça baigne (pas + jamais)

d. ça craint (plus + jamais + pas encore)

e. ça me dit (pas + plus)

S'il est possible de répondre négativement à la question ça boume? par le simple adverbe de négation non, il n'est pas possible de reprendre tout l'énoncé sous forme négative.

La négation et l'insertion d'un modifieur sont ainsi deux tests permettant de révéler qu'une locution ne peut supporter de modifications et que ça, sujet unique de certains verbes (ça barde, ça baigne) fait partie intégrante de la locution. En revanche, des expressions comme ça craint peuvent, elles, admettre des transformations syntaxiques. Qu'est-ce qui caractérise alors une construction comme ça craint, qui reste à la limite des expressions figées?

\subsection{Une construction quasi-impersonnelle : ça craint}

Si l'on prend l'exemple de ça craint, on ne peut pas parler, à son propos, de locution puisqu'un référent nominal est identifiable dans le cadre de la dislocation. Pour autant, une reprise en ça n'a pas la même valeur que la reprise par un pronom personnel (ça craint, ton devoir vs il craint, ton devoir). Ça apparait comme un sujet quasiment vide au niveau sémantique et il serait plus juste de qualifier ces expressions d'impersonnelles plutôt que de parler de locutions.

En définitive, il semble que ça craint ne soit pas une locution (composée du verbe craindre et du pronom ça) mais qu'il existe deux lexies craindre, dont une a le sens de redouter $^{2}$ (avec une construction de type $X$ craindre $Y$ ) et l'autre qui a le sens de : être insuffisant, minable, ne pas être à la hauteur qui admet un sujet quasi-impersonnel, à savoir le pronom ça.

Cette deuxième lexie craindre, répertoriée dans le dictionnaire comme sens II (familier) du verbe, a des contraintes syntaxiques propres. En plus d'avoir comme sujet privilégié ça (qui est un sujet factice mais obligatoire), cette lexie admet un argument en séquence (nous reprenons le terme d'argument en séquence, emprunté à Corblin 1995), qui peut être une infinitive ou une complétive, construction qui n'est pas possible pour ça barde:

\footnotetext{
${ }^{2}$ Nous reprenons les synonymes donnés par Le Petit Robert. Celui-ci établit en effet un sens I de craindre, transitif et un sens II, intransitif, qualifié de familier.
} 
(10) a. ça craint de voyager seul

b. ça craint que tu lui aies dit

c. * ça barde que tu fasses cela

d. * ça baigne de partir au soleil

Un autre test permettant de faire la différence entre ça craint (forme impersonnelle d'un verbe) et une locution comme ça barde, est celui de l'interrogation. On ne peut soumettre le sujet d'une locution à interrogation (* qu'est-ce qui est cuit? les carottes /* qu'est-ce qui barde? ça). Si l'interrogation est possible, comme pour ca craint (qu'est-ce qui craint? ça) c'est bien que le sujet n'appartient pas à une locution car il reste référentiel.

Dans ça craint, le pronom ça peut-être qualifié de quasi-impersonnel, comme nous l'annoncions en titre, et non d'impersonnel car, s'il est un sujet factice qui se double d'un argument en séquence, il conserve une trace de référentialité, ce qui n'est pas le cas de il impersonnel. Comme le dit Corblin (1994 : 45) :

Je défendrai en revanche l'idée qu'il n'y a pas de ça impersonnel en français moderne. Ce qui est perçu comme une concurrence dans la fonction de sujet impersonnel, me parât en réalité être une dualité de construction: la construction impersonnelle marquée par $i l$, et la construction à sujet indistinct réalisée par ça.

Pour une distinction plus fine entre ça et il impersonnel (qui n'est pas l'objet de cet article) nous renvoyons à Cadiot (1988) et Corblin (1994 et 1995). Mieux vaut donc parler, en suivant Corblin, de sujet quasi-impersonnel à propos de ça.

Pour résumer : quelle différence y a-t-il entre construction quasi-impersonnelle et construction figée ? C'est précisément dans la distinction de deux constructions que se trouve la réponse. Dans un premier cas, nous avons voulu démontrer qu'une construction figée en ça $V$, structure non modifiable, existe pour un nombre limité de verbes alors que dans un second cas, une construction en ça $V$ que $P / d e+i n f$ est possible pour un grand nombre de verbes, pour lesquels le démonstratif n'est qu'un sujet « vide » annonçant le sujet appelé « réel » par les grammaires traditionnelles.

Si une distinction fondée sur des critères syntaxiques et sémantiques est dès lors possible entre des locutions et des constructions impersonnelles, à travers les exemples de ça barde vs ça craint, et qu'il est possible de dégager des propriétés pour chacune de ces constructions, nous voudrions à présent établir une autre comparaison entre les constructions en ça $V$ et les constructions en se $V$ pour démontrer l'influence que peut avoir le pronom ça sur le verbe.

\section{Le rôle de ça sur certains verbes : un parallèle entre les constructions en ça $V$ et les constructions en se $V$}

Même si l'on ne peut pas véritablement parler de locution pour ça craint comme on peut le faire pour ça barde, on aperçoit un début de figement, dans ce type de construction, dû au rôle joué par ça puisque l'utilisation du démonstratif bloque la construction du verbe, comme le fait, d'une certaine manière le pronom se. 


\subsection{Le pronom ça contraint la construction du verbe}

Si l'on reprend l'exemple de ça craint, on perçoit que le démonstratif agit sur le verbe en ce sens qu'il permet de révéler le sens II du verbe craindre, donné par le dictionnaire. L'utilisation de ça ne fait alors que mettre en lumière la seconde lexie craindre et opère la distinction entre deux lexies, ce que nous évoquions précédemment.

Mais cette conséquence, d'ordre lexicologique, n'est pas la seule conséquence de l'association de ça et du verbe craindre. Il existe une influence plus décisive du démonstratif sur le verbe. Observons les exemples suivants :

(11) a. Marie craint les chiens

b. * ça craint les chiens

c. ça craint

Les exemples (11) font ressortir la construction de base du verbe craindre (lexie $1=$ redouter) qui est : $X$ craindre $Y$, c'est-à-dire une construction à deux actants. Or, si l'on remplace le sujet Marie par ça, on choisit la deuxième lexie craindre (lexie $2=$ être minable) et on s'aperçoit que l'emploi transitif du verbe n'est plus possible (une construction possible serait : ça craint, les chiens! mais il s'agirait alors d'une structure disloquée, perceptible à l'écrit par la ponctuation et à l'oral par l'intonation, structure qui est bien différente de celle envisagée en (11b). Dans cette autre acception de craindre, le pronom ça fait partie du régime de craindre mais le verbe est alors intransitif. On peut donc dire que de même que ça révèle un autre sens de craindre, l'utilisation du démonstratif a des conséquence sur la construction du verbe.

Prenons un autre exemple montrant la modification de la valence opérée par le pronom ça. Dans l'exemple ça vend bien, l'actant sujet se fond dans un ça qui n'évoque plus une personne distincte. Par ce phénomène, on insiste sur le procès et seul compte le fait de signaler qu'il y a de la vente. Qui vend et ce qui est vendu importe peu. Si l'on compare cette construction avec d'autres, dont le sujet est non identifiable, on constate que ça vend bien est la construction la plus radicale. Observons l'évolution actantielle à l'œuvre dans les exemples suivants :
a. on vend bien les livres ici
b. ça se vend bien, les livres, ici
c. les livres se vendent bien
d. ça vend bien ici
e. * ça vend bien les livres ici
f. les étudiants travaillent la linguistique
g. ? ça travaille la linguistique
h. ca travaille ici

Le pronom indéfini on (12a) est imprécis quant à la référence qu'il induit (on ne sait pas qui est le vendeur) mais indique la présence d'un agent humain ; le verbe garde sa structure de base à deux arguments ( $X$ vendre $Y$ ). En (12b), la trace d'un agent humain est conservée mais amoindrie par ça et la voix moyenne introduite annonce 
déjà l'idée que le procès se réalise sans l'intervention d'un agent précis. En revanche, la voix moyenne utilisée seule en (12c) supprime l'un des actants. Mais (12d) va plus loin en supprimant les deux actants pour insister sur le procès exprimé par le verbe. Le verbe qui était transitif ne l'est plus et c'est donc sa valence qui est altérée. L'analyse serait la même pour ça travaille ici : seule l'action de travailler compte ; le sujet et l'objet ont disparu pour ne garder que l'indication du procès. On a donc l'apparition d'une construction impersonnelle en $c ̧$, d'un genre particulier.

Si nous souhaitons établir un parallèle entre les constructions en ça $V$ et les constructions en se $V$, c'est parce qu'on assiste aux mêmes effets (révélateur de lexies différentes et modification de la valence du verbe, à des degrés divers) pour les tournures en $s e V$, du fait de l'utilisation du pronom se.

\subsection{Un même rôle joué par le pronom se}

Une brève comparaison, à l'issue de cet article, entre le rôle joué par ça sur certains verbes (comme craindre) et le rôle joué par se, permet une ouverture sur le type de modifications opérées par certains pronoms, sur un verbe. Prenons l'exemple de douter et se douter :

(13) a. je doute qu'il vienne

b. je me doute qu'il viendra

Le verbe douter employé seul indique l'état d'incertitude tandis que le même verbe associé au pronom se, signifie presque le contraire (la quasi certitude). On voit que l'emploi du pronom se révèle un glissement de sens substantiel ainsi que l'existence de deux lexies (douter/se douter). C'est le même phénomène (la différence entre deux lexies) que faisait déjà apparaitre ça, associé au verbe craindre.

Mais l'emploi de se a d'autres conséquences que celui de révéler la seconde lexie d'un verbe. De même que ça peut, dans certains cas, modifier la valence du verbe, se peut également contraindre la construction du verbe et «figer» le verbe à des degrés divers.

La construction en $s e V$ englobe différents types de verbes, réfléchis, réciproques, médio-passifs ou qui n'existent qu'à la voix moyenne (Creissels 1995). La construction de ces verbes avec le pronom se s'éloignent plus ou moins de la forme simple, selon les cas. Observons divers exemples :

(14) a. elle se lave

b. elle s'aperçoit de son erreur

c. elle se sowvient

L'exemple (14a) utilise la forme réfléchie de laver: sémantiquement, on a toujours une construction à deux actants $(X$ laver $Y / X)$ et le pronom se a un référent nominal mais syntaxiquement, le verbe devient monovalent et intransitif. En (14b) en revanche, on assiste à une modification de la valence par rapport au verbe apercevoir ( $X$ apercevoir Y). Se n'a plus de co-référent (comme pour 14a) et le verbe apercevoir qui était transitif, devient transitif indirect (s'apercevoir de). Enfin, se sowvenir va plus loin encore car la forme souvenir n'existe plus si elle n'est pas associée au pronom se. 
On observe alors les mêmes modifications que celles que mettait en évidence ça et l'apparition de plusieurs lexies, que révèle l'emploi du pronom se. Dans ce cas, la construction du verbe se modifie en fonction de l'emploi du morphème se. A travers ces différents exemples, on peut émettre l'idée qu'il existe des degrés de figement dans les constructions en se $V$ comparables à ceux qu'on observait précédemment pour les constructions en $c a V$. Cette idée légitime notre rapprochement final.

\section{Conclusion}

Le but de cette étude n'était pas de dresser une liste non exhaustive des expressions figées qui ont ça pour sujet. Il serait peut-être même vain de le faire puisque le langage produit sans cesse de nouvelles tournures avec $c ̧$. Notre objectif était plutôt de faire la différence entre des expressions comme ça me fait une belle jambe, ça rime à rien, ça craint, ça barde, que l'on regroupe généralement sous l'appellation de construction figée, sans chercher à faire de distinction entre elles. Or, nous avons essayé de montrer que ces expressions sont différentes selon le rôle que joue ça (un sujet possible, un sujet privilégié ou le seul sujet possible d'un verbe) et qu'on ne peut à proprement parler de locution que pour un petit nombre de constructions (comme ça barde).

De plus nous avons tenté de montrer que si ça peut certes être le sujet figé d'une locution, il peut aussi être un sujet quasi-impersonnel qui contraint la construction d'un verbe. Il a un rôle à la fois lexicologique (celui de faire apparaitre une lexie du verbe) et syntaxique (il modifie parfois la construction du verbe). C'est ce que nous avons essayé de démontrer à partir de l'exemple de craindre. Il peut être comparable, en ce sens, au pronom se dans les constructions pronominales. Ce double rôle (sémantique et syntaxique) d'un pronom sur le verbe justifie le rapprochement final et la progression de cet article : de ça barde à se souvenir en passant par ça craint.

Le figement n'est pas seulement alors un problème lexical; il peut être lié à l'utilisation d'un pronom qui va orienter la construction du verbe et sélectionner une lexie du verbe. Il conviendrait alors, pour poursuivre ce raisonnement, d'étudier les différentes classes de verbes qui sont associés à ça et qui commandent peut-être l'apparition d'un tel pronom. 
Expression compositionnelle ou locution : 'ça craint' vs 'ça barde'?

\section{BIBLIOGRAPHIE SOMMAIRE}

Anscombre, J.-C., (1998), «ça, c'est quelque chose. Quelques caractéristiques de la reprise d'un groupe nominal par ça/ce », Recherches en Linguistique et Psychologie cognitive, 9, pp. 83-105.

Blanche-Benveniste, C. et alii, (1987, 2e edition), Pronom et syntaxe, l'approche pronominale et son application au français, Paris, SELAF.

CADiot, P. (1988), «De quoi ça parle ? A propos de la référence de ça, pronom-sujet», Le français moderne $\mathrm{n}^{\circ} 56$, octobre 88 , Paris, CNRS.

CORBLIN, F. (1994), «Existe-t-il un «ça » impersonnel en français ? », L'information grammaticale, $\mathrm{n}^{\circ} 62$, pp. $45-47$.

CORBLIn, F. (1995), Les formes de reprise dans le discours, Anaphores et chaînes de référence, Rennes, Presses Universitaires de Rennes.

Creissels, D. (1995), Eléments de syntaxe générale, Paris, Presses Universitaires de France.

Fiala, P., LAfON, P. et Piguet, M.-F., (1997), La locution : entre lexique, syntaxe et pragmatique, Paris, Klincksieck, publication de l'INALF, collection « Saint-Cloud».

Gross, G. (1996), Les expressions figées en français, Paris, Ophrys.

Gross, M. (1982), «Une classification des phrases «figées» du français », Revue québécoise de linguistique, 11, 2, pp. 151-185.

Gross, M. (1993), «Les phrases figées en français », L'information grammaticale, 59, pp. 36-41.

Maillard, M. (1989), Comment ça fonctionne, thèse de linguistique française pour le doctorat d'Etat, sous la direction de J. Dubois, Paris X.

MejRI, S. (2003), «Polysémie et polylexicalité », Syntaxe et Sémantique, 5, Caen, Presses Universitaires de Caen.

MejRI, S. (2002), «Le figement lexical: nouvelles tendances», Cabiers de lexicologie, 80, 1, pp. 213-225.

Mel'Čuk, I., Clas, A., POlguere, A., (1995) Introduction à la lexicologie explicative et combinatoire, Louvain-la-Neuve, Duculot.

Olsson, H. (1986), La concurrence entre il, ce et cela (ça) comme sujet d'expressions impersonnelles en français contemporain, Stockholm, Almqvist et Wiksell.

Polguere, A. (2003), Lexicologie et sémantique lexicales, Montréal, Presses Universitaires de Montréal.

Rey, A. et Chantreau, S. (1997), Dictionnaire d'expressions et locutions, Paris, Le Robert.

TOLLIS, F. (2001), La locution et la périphrase : du lexique à la grammaire, Paris, L'Harmattan. 
